\title{
Diseño de centros educativos públicos preuniversitarios en Galicia (2008-2017): ¿inclusivos y sostenibles?
}

\section{The design of public schools in Galicia (2008-2017): inclusive and sustainable?}

\author{
Santiago Eduardo Pato Rodríguez* \\ * Universidade de Vigo
}

\begin{abstract}
Resumen
En este trabajo se revisan los criterios de diseño de los centros educativos públicos de enseñanzas preuniversitarias de la Comunidad Autónoma de Galicia durante la última década (2008-2017) bajo el prisma de la inclusión educativa y el desarrollo sostenible. Para ello se han estudiado las condiciones técnicas que la Administración Educativa Gallega exige a los proyectos de construcción de estos centros mediante un análisis de contenido de los contratos licitados en el período señalado. A partir del estudio realizado, se concluye que apenas se exigen premisas técnicas de diseño que favorezcan la inclusión educativa o el desarrollo sostenible.

Palabras clave: [Ambiente Escolar, Entorno Construido, Educación Inclusiva, Desarrollo Sostenible]
\end{abstract}

\begin{abstract}
In this paper we review the design criteria of public schools of Galicia during the last decade (2008-2017) under the prism of educational inclusion and sustainable development. In order to do this, the technical conditions that the Galician Educational Administration requires to the construction projects of these schools have been studied through a content analysis of the contracts tendered in the indicated period. From the study carried out, it is concluded that barely technical design premises are required that encourage educational inclusion or sustainable development.

Keywords: [School Environment, Built Environment, Inclusive Education, Sustainable Development]
\end{abstract}

\section{Introducción}

En España, con arreglo a la Ley de Contratos del Sector Público (Real Decreto Legislativo 3/2011, de 14 noviembre, por el que se aprueba el texto refundido de la Ley de Contratos del Sector Público. Texto consolidado), el primer paso en el proceso de construcción de centros educativos públicos consiste en la publicidad que realiza la Administración Educativa para contratar el proyecto constructivo (entendido como contrato de servicios). En esta fase, la Administración fija los criterios y condiciones administrativas, técnicas y económicas para que estudios privados de arquitectura presenten sus ofertas a fin de resultar adjudicatarios del proyecto de construcción. Las condiciones administrativas y económicas impuestas por la
Administración se fijan en el Pliego de cláusulas administrativas, mientras que las exigencias técnicas se recogen en el Pliego de prescripciones técnicas. Ahora bien, la Administración contratante se reserva el derecho de publicar en esta fase, además de los citados, otros documentos que recojan cuestiones de obligado cumplimiento. Una vez presentadas las ofertas, la Administración procede a su evaluación para, a continuación, adjudicar el proyecto constructivo a la oferta con mejor valoración a partir de los criterios previamente fijados. En el caso particular gallego, la información y documentación relativa a estos procedimientos de contratación se puede consultar desde el año 2008 en la Plataforma de Contratos Públicos de Galicia: www.contratosdegalicia.gal/portada.jsp.

Por otra parte, el fomento de la inclusión y la sostenibilidad desde los centros educativos es una cuestión clave en la que diversos autores ya se han manifestado: Booth y Aiscow, en su Index for Inclusion (2011), señalan explícitamente que "los centros escolares que se desarrollan inclusivamente son lugares que fomentan un desarrollo sostenible" (p. 24), destacando que "es fundamental para la inclusión la sostenibilidad medioambiental" (p. 24); en el ámbito nacional, Echeita y Navarro (2014) indican que "sólo a través de una educación para la sostenibilidad y la inclusión estaremos en condiciones de capacitar a todos los estudiantes para llevar el tipo de vida que consideran valioso e incrementar sus posibilidades reales de elección” (p. 159). Análogamente, estos autores destacan que "una educación para la sostenibilidad y para la inclusión es necesaria, incluso urgente" (p. 158).

En este sentido, es fundamental acometer una transformación de nuestras instituciones educativas: en 2010 se celebra en Madrid la Conferencia Internacional "La Educación Inclusiva: vías para favorecer la cohesión social" donde se subraya la importancia de transformar los centros educativos para avanzar hacia la educación universal, reclamando para ello "eliminar todo tipo de barreras (físicas, de acceso al currículo, actitudinales, tecnológicas, de socialización y comunicación)" (European Agency for Development in Special Needs Education, 2010, p.3). En esta necesaria 
transformación de los centros, la optimización de sus características físicas se torna indispensable. La literatura existente señala la necesidad de "disponer de una organización de centro y de aula que hagan viable y funcional la atención educativa a todo el alumnado, adaptándola a las diferencias individuales" (Jiménez \& García, 2013, p. 233). Otros estudios de calado también insisten en la necesidad de mejorar la equidad en las escuelas considerando las mismas en su entorno socio-educativo más amplio en que se encuentran (Parrilla, Susinos y Gallego, 2014). De este modo, se consideran clave las siguientes cuestiones: accesibilidad, participación, flexibilidad/adaptabilidad de los centros y relación con el entorno.

Con respecto a la sostenibilidad, en los últimos años han aparecido indicadores con metodologías específicas asociadas que permiten cuantificar de forma científicamente fidedigna el carácter sostenible o no sostenible de cualquier sistema u organización humana, tomando en consideración todas las posibles categorías de consumo, además de ocupación de suelo y generación de residuos. Cabe destacar, en este sentido, la metodología MC3 (Doménech, 2004), que divide las posibles fuentes de emisión en 9 grandes grupos o secciones de consumo: emisiones directas (combustibles y otras emisiones directas), emisiones indirectas (electricidad y otras emisiones indirectas), materiales no orgánicos, servicios y contratos (servicios de movilidad y comunicaciones), recursos agropecuarios, recursos forestales, huella del agua (consumo de agua potable y no potable), uso del suelo y residuos, vertidos y emisiones.

Pues bien, este trabajo tiene por objeto estudiar el grado de consideración que tienen tanto la inclusión como el desarrollo sostenible en las premisas de diseño de los centros educativos públicos de enseñanzas preuniversitarias de la Comunidad Autónoma de Galicia durante la última década (desde 2008 hasta 2017). De este modo, se delimita el estudio a las características físicas del centro, correspondientes, siguiendo a González, Nieto y Portela (2003) a su dimensión estructural (específicamente, estructuración material: espacios, recursos e instalaciones), a partir de la cual se pescudan los criterios de inclusión y sostenibilidad tenidos en cuenta en el diseño de los centros estudiados por la Administración educativa.

Para alcanzar dicho fin, el estudio se centra en la revisión de la información de los contratos licitados por la Administración Educativa Gallega. Para ello, se realiza específicamente un análisis documental de los pliegos de condiciones exigidas por la Administración en la fase de publicidad para contratar el proyecto constructivo.

\section{Metodología}

Desde 2008 se ha contratado en Galicia el diseño y construcción de 10 nuevos centros públicos (una Escuela de Educación Infantil (en lo sucesivo EEI), 5 Colegios de Educación Infantil y Primaria (en lo sucesivo CEIP) y 4 Institutos de Educación Secundaria, en adelante IES).
Los pasos seguidos en el presente estudio han sido:

1) Revisión y selección de la información pública referida en la plataforma digital de contratación de la Xunta de Galicia: en primer lugar, se ha revisado el inventario completo de contratos licitados desde 2008 por la Administración Educativa Gallega (Consellería de Cultura, Educación e Ordenación Universitaria): 575 procedimientos. Seguidamente se seleccionaron los procedimientos relativos a la construcción de nuevos centros educativos de nivel preuniversitario, detectando 10 casos, que se señalan a continuación (entre paréntesis: provincia de localización del centro y año de publicación de las condiciones exigidas por la Administración para los proyectos constructivos):
a. $\quad$ CEIP en Boiro (A Coruña, 2009)
b. $\quad$ CEIP en Oroso (A Coruña, 2009)
c. IES en Milladoiro (A Coruña, 2009)
d. IES en Culleredo (A Coruña, 2010)
e. CEIP en Oleiros (A Coruña, 2011)
f. IES en Soutomaior (Pontevedra, 2012)
g. $\quad$ CEIP en Culleredo (A Coruña, 2015)
h. CEIP en Mesoiro (A Coruña, 2016)

Es necesario señalar que también se encontraron procedimientos relativos a una EEI en Betanzos y un IES en Carral (ambos en la provincia de A Coruña) para los que no existe documentación en la plataforma digital de contratos sobre la publicidad de las condiciones exigidas para sus proyectos constructivos.

2) Recopilación de la información relativa a los contratos de licitación de los proyectos de construcción de los centros señalados: se reúnen los documentos publicados por la Administración educativa en los que ésta dicta las condiciones prescriptivas que ha de cumplir el diseño de estos centros. Básicamente, se trata de dos documentos para cada caso:

a. Pliego de Cláusulas Administrativas Particulares (en adelante PCAP)

b. Pliego de Prescripciones Técnicas Particulares (en adelante PPTP)

No obstante, para los tres últimos casos, la Administración publica un documento prescriptivo adicional denominado "Normas de diseño y constructivas de los edificios de uso docente", de notable interés para este estudio.

3) Análisis de contenido de los documentos reunidos: partiendo de las categorías "inclusión" y "sostenibilidad", se ha acometido un análisis documental centrado en las condiciones técnicas impuestas por la Administración para el diseño de las características físicas de los centros susceptibles de mediar en los procesos educativos. En total se han analizado 18 documentos:

a. CEIP en Boiro: PPTP (no se encuentra publicado su PCAP).

b. CEIP en Oroso: PCAP y PPTP.

c. IES en Milladoiro: PCAP y PPTP.

d. IES en Culleredo: PCAP y PPTP.

e. $\quad$ CEIP en Oleiros: PCAP y PPTP.

f. IES en Soutomaior: PCAP, PPTP y

"Normas de diseño y constructivas de los edificios de uso docente" 
g. CEIP en Culleredo: PCAP, PPTP y "Normas de diseño y constructivas de los edificios de uso docente"

h.

CEIP en Mesoiro: PCAP, PPTP y "Normas de diseño y constructivas de los edificios de uso docente"

Conviene señalar que se ha considerado pertinente incluir en el análisis los PCAP porque también recogen premisas técnicas relevantes como los criterios de valoración técnica de las ofertas.

Para la ejecución del análisis se han seguido las recomendaciones sugeridas por Bardin (1986) y López (2002). Así, para cada documento analizado se identifican las unidades de información relativas a las categorías de partida.

Además, dentro de cada categoría y siguiendo el corpus teórico incluido en la introducción, se han considerado las siguientes subcategorías:

-Inclusión: accesibilidad, participación, adaptabilidad/flexibilidad, relación con entorno.

-Sostenibilidad: se han considerado las categorías que comprende la metodología MC3 realizando las siguientes simplificaciones: se han unido en una única categoría (Energía) las emisiones directas e indirectas; se han unido en una única categoría (materiales) los materiales no orgánicos, los recursos agropecuarios y los recursos forestales. Así, se consideran las siguientes 6 subcategorías de análisis: energía, materiales, transporte y comunicaciones, agua, uso del suelo y residuos.

\section{Resultados}

Se incluyen a continuación los hallazgos más relevantes de la investigación realizada.

\section{Pliegos de Prescripciones Técnicas Particulares}

Estos documentos se centran fundamentalmente en cuestiones de forma del proyecto. Comprenden el contenido mínimo de los documentos de los proyectos y forma de presentación de la documentación. Además, en todos ellos se indica que "los trabajos presentados (proyecto de ejecución) se realizarán de acuerdo con las disposiciones de la se realizarán de acuerdo con las disposiciones de la Parte I del Código Técnico de la Edificación y su Anexo I "Contenido del Proyecto", aprobado por el Real Decreto 314/2006, de 17 de marzo (BOE del 28 de marzo de 2006)". Ahora bien, se incluye en todos los casos una tabla con la especificación de espacios, metros cuadrados y costes que la Administración Educativa requiere para cada proyecto particular. En estas especificaciones siempre se contempla un porcentaje del $25 \%$ del total de superficie útil de espacios para circulaciones, cuestión que se enmarca dentro de la categoría Inclusión y subcategoría Accesibilidad. Además, cabe destacar las siguientes particularidades recogidas en las citadas tablas para cada caso, todas ellas relativas a la categoría Inclusión y subcategoría accesibilidad a excepción de una unidad de información detectada en el IES de Soutomaior:

1) CEIP en Boiro y CEIP en Oroso: en ambos casos se contempla la previsión de aseos para personas con discapacidad: "SERVICIOS COMUNES: Anexo
Aseos (minusválidos): $m 2$ del espacio: 6; número de espacios: 2; total m2: 12" (p. 4)

2) IES en Milladoiro e IES en Culleredo: a diferencia del resto de casos, no se contempla específicamente una previsión de dotación de espacios para aseos para personas con discapacidad.

3) CEIP en Oleiros: aunque no se contempla específicamente dotación de espacios para aseos para personas con discapacidad, sí se contempla una previsión de espacios para anexo de aseos: "ZONA DOCENTE: Anexo aseos: $m 2$ del espacio: 6; número de espacios: 1; total m2: 6" (p. 16).

4) IES en Soutomaior: se contempla la previsión de aseos para personas con discapacidad: "ZONA DOCENTE: Aseos (minusválidos): $m 2$ del espacio: 6; número de espacios: 1; total m2: 6" (p. 16). Además, también se contempla desde la Administración educativa la dotación de espacio para un depósito de combustible, indicador de que se opta por un sistema de calefacción de combustión; desechando de antemano otras posibilidades más sostenibles: "ZONA $D E$ INSTALACIÓN: Cuarto depósito combustible: $m 2$ del espacio: 15; número de espacios: 1; total m2: 15" (p. 17). (categoría: Sostenibilidad; subcategoría: Energía)

5) CEIP en Culleredo y CEIP en Mesoiro: estos dos casos son los que presentan una dotación más detallada de espacios para personas con discapacidad, a saber: "ZONA DOCENTE: Aseos alumnos primaria (con minusválidos): total m2: 90" (p. 14); "ZONA DOCENTE: Gimnasio, vestuarios (con minusválidos), despacho con aseo y almacén: total m2: 240" (p. 14); "ZONA DE ADMINISTRACIÓN: aseos profesores y visitas (con minusválidos): total m2: 15" (p. 14) .

\section{Pliegos de Cláusulas Administrativas Particulares}

Estos pliegos recogen la definición del régimen de contratación, presupuesto, licitación, adjudicación y ejecución del contrato. Ahora bien, también incluyen en todos los casos los criterios de valoración $\mathrm{y}$ adjudicación de las propuestas (puntuados sobre 100), de interés en el estudio acometido puesto que en estos criterios se manifiestan las prioridades de la Administración a la hora de elegir el proyecto que finalmente se construirá:

1) CEIP en Oroso e IES en Milladoiro: en ambos casos se contemplan los siguientes criterios de valoración de las propuestas: "Volumetría e integración (hasta 20 puntos): armonía y proporcionalidad de la propuesta e integración en el contorno" (p. 9). Se considera que este criterio guarda cierta relación con la subcategoría Relación con entorno dentro de la categoría Inclusión; “Adaptación funcional y técnica del edificio (hasta 23 puntos): funcionalidad educativa del edificio ya sea en sus aspectos de circulaciones o sectorización del inmueble, así como también la adaptación de la idea propuesta a un desarrollo constructivo adecuado" (p. 9). Se considera que este criterio guarda relación con la subcategoría Accesibilidad (categoría Inclusión). Ahora bien, los demás criterios de valoración son legibilidad gráfica (calidad de la documentación gráfica presentada y claridad de expresión (hasta 5 puntos)), plazo de 
ejecución (máxima puntuación hasta 5 puntos a la propuesta que oferte el plazo menor) y valoración económica (hasta 47 puntos).

2) IES en Culleredo: en este caso, aunque con una mínima mayor especificación, los criterios de valoración y su puntuación son análogos a los anteriores.

3) CEIP en Oleiros e IES en Soutomaior: en estos dos casos se otorga un peso mayoritario al precio ofertado de cara a la adjudicación (65 puntos sobre 100). Las cuestiones técnicas se valoran así: "Integración del edificio (20 puntos): adaptación a la parcela (unidad de información encuadrada dentro de la categoría inclusión: relación con entorno); obtención de volumetrías adaptadas a la tipología de uso escolar con coste de ejecución adecuado" (p.21) (unidad de información encuadrada dentro de la categoría inclusión: adaptabilidad). "Adaptación funcional y técnica (12 puntos): se valorará la adaptación de la propuesta al programa de necesidades aprobado por la Consellería de Educación y Ordenación Universitaria" (p. 21) (unidad de información encuadrada dentro de la categoría inclusión: adaptabilidad). Por último se otorga una valoración residual de hasta 3 puntos a mejoras en la documentación tales como maquetas, vistas fotorrealistas o 3D.

4) CEIP en Culleredo y CEIP en Mesoiro: al igual que los casos anteriores, el mayor peso en la puntuación de los criterios de adjudicación se lo lleva el precio ofertado (hasta 60 puntos). Los criterios técnicos se valoran así: "Integración del edificio (hasta 18 puntos): adaptación del edificio a los condicionantes externos del entorno e internos de la parcela, así como la adecuación de la volumetría y materiales empleados" (p. 3). A su vez, se desglosa de la siguiente manera:

a. "Adaptación al entorno (hasta 5 puntos)", (p.3); unidad de información encuadrada dentro de la categoría inclusión: relación con entorno.

b. "Adaptación a la topografía de la parcela (hasta 5 puntos)" (p.3); unidad de información encuadrada dentro de la categoría inclusión: relación con entorno.

c. "Volumetría adaptada al uso del edificio (hasta 4 puntos)" (p.4); unidad de información encuadrada dentro de la categoría inclusión: adaptabilidad.

d. "Idoneidad en materiales empleados: economía, durabilidad, estética (hasta 4 puntos)" (p.4); unidad de información encuadrada dentro de la categoría Sostenibilidad: materiales.

“Adaptación funcional y técnica (hasta 18 puntos): en este apartado se valora el esquema distributivo del edificio acomodado al uso, así como a la adaptación de la propuesta en superficie construida y presupuesto al previsto en el programa de necesidades" (p. 4). Se desglosa específicamente dotando de hasta 12 puntos "la adaptación al uso del edificio y cumplimiento de la normativa técnica y urbanística" (p. 4); unidad de información encuadrada dentro de la categoría inclusión: adaptabilidad; contemplando también "la adaptación en superficie construida al programa (hasta 4 puntos) y la adaptación y justificación del avance de presupuesto (hasta 2 puntos)", (p. 4).
Por último se otorga un valor residual de hasta 4 puntos a la calidad, legibilidad y claridad en la expresión de la idea.

Asimismo, y aunque no relacionado con las características físicas del centro, sí que resulta interesante destacar cómo en todos los PCAP se contempla como criterio de desempate en la adjudicación la existencia de un plan de igualdad en la empresa. Además, desde el año 2010, también se contempla como primer criterio de desempate que el cuadro de personal de la empresa cuente con un número de trabajadores con discapacidad no inferior al 2 por 100 .

Normas de diseño y constructivas de los edificios de uso docente

Desde el año 2012 la Administración Educativa incluye este documento dentro de las condiciones prescriptivas que ha de cumplir el diseño de centros educativos. Se trata de una guía de 52 páginas elaborada por el Servicio de Supervisión de Proyectos y Gestión Patrimonial de la Consellería de Cultura, Educación y Ordenación Universitaria, con el fin de facilitar la elaboración de proyectos de Centros Públicos y como complemento a todas las normativas de rango superior y obligado cumplimiento que se encuentren en vigor cuando se redacte el proyecto.

La versión analizada de este documento es la publicada para el CEIP de Mesoiro (2016), puesto que, además de ser la última versión disponible, es la misma que para el CEIP de Culleredo (2015) y una versión actualizada de la del IES Soutomaior (2012), contemplando, de este modo, algunas cuestiones adicionales y suprimiendo otras. En todo caso, las diferencias con la versión publicada para el IES Soutomaior no son relevantes para el estudio acometido.

En la tabla que se muestra a continuación se incluye un resumen del análisis acometido. 
Tabla 1.

Resumen análisis de contenido del documento "Normas de diseño y constructivas de los edificios de uso docente"

\begin{tabular}{|c|c|c|c|}
\hline Categoría & Subcategoría & $\begin{array}{c}\text { Uds. de } \\
\text { información }\end{array}$ & Total \\
\hline \multirow[t]{5}{*}{ INCLUSIÓN } & General & 1 & \multirow{5}{*}{29} \\
\hline & Accesibilidad & 22 & \\
\hline & Participación & 2 & \\
\hline & $\begin{array}{l}\text { Adaptabilidad } \\
\text { /flexibilidad }\end{array}$ & 2 & \\
\hline & $\begin{array}{l}\text { Relación con } \\
\text { entorno }\end{array}$ & 2 & \\
\hline \multirow[t]{7}{*}{ SOSTENIBILIDAD } & General & 2 & \multirow{7}{*}{32} \\
\hline & Energía & 12 & \\
\hline & Materiales & 10 & \\
\hline & $\begin{array}{l}\text { Transporte y } \\
\text { comunicaciones }\end{array}$ & 1 & \\
\hline & Uso de suelo & 5 & \\
\hline & Agua & 2 & \\
\hline & Residuos & 0 & \\
\hline
\end{tabular}

En primer lugar cabe destacar cómo estas normas se centran, en lo relativo a inclusión, en cuestiones de accesibilidad: dentro de las generalidades que han de reunir los centros se indica explícitamente que "todos los centros deberán ser accesibles a discapacitados en todas las plantas del edificio" (p. 4). Asimismo, se especifican aspectos físicos de los espacios escolares que superan los mínimos exigidos por normas tales como el Código Técnico de la Edificación o el Reglamento de desarrollo y ejecución de la ley de accesibilidad y eliminación de barreras en Galicia (DOG $\mathrm{n}^{\circ}$ 41, de 29 de febrero de 2000). A modo de ejemplo, en estas normas se indica que la altura libre en espacios docentes "será por lo menos de 3 metros" (p. 5) y el ancho de los pasillos será de "un mínimo de 2 metros" (p. 5) y en el citado Reglamento, los mínimos exigidos son, respectivamente, 2,20 metros y 1,80 metros. En las normas también se encuentran numerosas referencias a la dotación de espacios totalmente accesibles para personas con discapacidad (aseos: p. 6, 7 y 11; secretaría: "en secretaría se dispondrá un mostrador adaptado a minusválidos", p. 23; ascensor: "El ascensor estará adaptado al uso de minusválidos", p. 38). Otras cuestiones consideradas dentro de la accesibilidad son la señalización y protección de espacios de circulación (escaleras, pasillos, espacios exteriores), la zona de aparcamiento (con una plaza adaptada "al uso de minusválidos", p. 48) y la especificación de elementos de uso habitual (puertas, ventanas, elementos de fontanería) para su uso por personas con discapacidad.

Asimismo, se detectan dos unidades de información relacionadas con el fomento de la participación en el centro: el cuidado del diseño de los espacios exteriores para "potenciar un uso más creativo y participativo de éstos" (p. 7) y la posibilidad de contar con un huerto escolar "cuando se ponga en uso el edificio" (p. 49).
Como premisas generales de diseño, además de indicar que la solución arquitectónica debe contribuir a "desarrollar en el alumnado hábitos de convivencia y de buena relación con el ámbito escolar" (p.3), se contempla la flexibilidad, en el sentido de adecuarse " $a$ las necesidades de escolarización concretas" (p. 4). Además, al hablar de la estructura de los centros se indica que "deberán proyectarse sobre la base de una retícula estructural modular que permita la máxima flexibilidad de redistribución de locales" (p. 13). Además, también se indica que "en los proyectos se tendrá en cuenta la buena integración en su ámbito urbano" (subcategoría: relación con entorno). La relación con el entorno también se subraya al hablar de la jardinería de los espacios exteriores, donde se recomienda introducir "especies vegetales dedicando una mayor atención hacia especies autóctonas o de mayor interés didáctico" (p. 49).

Con respecto a la categoría Sostenibilidad, existe una mayor variabilidad en la distribución temática de las unidades de información detectadas. Ya en las generalidades de las normas de diseño se indica que "en la concepción del proyecto debe presidir un principio de funcionalidad y economía" (p. 3). También destaca la sensibilidad presente en el documento hacia el fomento de actitudes sostenibles (se subraya el especial cuidado que ha de tener el proyectista al diseñar los espacios libres de edificación: su "tratamiento debe reflejar el respeto del hombre por su ámbito inmediato, necesario para alcanzar un mayor desarrollo de la sensibilidad de educadores y alumnos por la defensa del medio", p. 7). No obstante, la mayor parte de las unidades de información corresponden a la categoría Energía, donde se insiste en varias ocasiones en la importancia de una adecuada iluminación natural y aislamiento. Además, también se contemplan las energías renovables ("en los centros educativos se promoverán las instalaciones de energías renovables", (p. 4); "se recomienda la instalación de termos acumuladores eléctricos con apoyo solar", (p. 27)) y la exigencia de "clasificación energética $A$ en las lámparas a instalar" (p. 32). Se encuentran también numerosas unidades de información relativas a los materiales, siempre contemplados desde las premisas generales de "adecuada durabilidad con gasto mínimo de conservación" (p. 3) y, más específicamente, incidiendo sobre su resistencia, aislamiento y seguridad.

Cabe destacar, en lo referente a la subcategoría "Uso del suelo", que ya en las generalidades de las normas de diseño (Título I), se destaca la importancia de la concentración de la edificación, pues "favorece la vigilancia, limpieza y conservación del edificio a la vez que reduce el gasto de mantenimiento de este" (p. 3). Asimismo, en el apartado destinado a las normas para jardinería, se subraya la importancia de la "introducción de especies vegetales" en los espacios exteriores para "favorecer el conocimiento de especies botánicas", se contempla la presencia de un huerto escolar y se priman los "elementos vegetales que gocen de un mantenimiento sencillo y económico" (p. 49).

Se encuentran unidades de información de interés relacionadas con la subcategoría Agua: como criterio 
general se indica que "se debe procurar la concentración horizontal y vertical de aseos $y$ laboratorios para conseguir recorridos mínimos de la red de agua y desagües" (p. 25) y la obligatoriedad de que los "grifos sean temporizados" (p. 26).

Por último, también se contempla, en relación a la subcategoría Transporte y comunicaciones la posibilidad de albergar un aparcamiento de motos y bicicletas. Si bien, se le da un tratamiento no prescriptivo: "se tratará de buscar un sitio, a ser posible cubierto, para el aparcamiento de motos y bicicletas" (p. 48).

Como nota adicional del análisis de contenido acometido, cabe indicar que en este documento se cita hasta en 9 ocasiones el término "minusválidos", en una ocasión los términos "personas con minusvalias" y "discapacitados" y sólo en dos ocasiones el término "personas con discapacidades".

\section{Conclusiones}

En primer lugar, y desde un análisis meramente terminológico, es indispensable señalar cómo hasta en los documentos más recientes se siguen empleando términos como "minusválido". En este sentido, es evidente que el conjunto de pliegos y normas estudiadas no prestan excesiva atención a un adecuado uso terminológico inclusivo. Cabe recordar que, en España, desde el 2007 se debe utilizar en textos normativos el término "persona con discapacidad" (Ley 39/2006, de 14 diciembre de Promoción de la Autonomía Personal y Atención a las personas en situación de dependencia, Disposición adicional octava).

Con respecto a los PPTP, es cierto que en todos ellos se hace referencia a que los proyectos se realicen de acuerdo con las disposiciones de la Parte I del Código Técnico de la Edificación (en lo sucesivo CTE) y su Anexo I "Contenido del Proyecto". Independientemente que el análisis en profundidad de este documento trasciende el presente estudio, sí que es relevante señalar que el CTE se limita a incluir generalidades sin acompañarse de premisas técnicas específicas. Los aspectos relativos a sostenibilidad se reducen a exigencias básicas de ahorro de energía (a modo de ejemplo: Artículo 15.3 de la Parte I del CTE: "Exigencia básica HE 3: Eficiencia energética de las instalaciones de iluminación: los edificios dispondrán de instalaciones de iluminación adecuadas a las necesidades de sus usuarios" (p. 13)) y de suministro de agua: "los edificios dispondrán de medios adecuados para suministrar al equipamiento higiénico previsto agua apta para el consumo de forma sostenible" (p. 12). Las referencias a la accesibilidad se limitan a indicar que "se facilitará el acceso y la utilización no discriminatoria, independiente y segura de los edificios a las personas con discapacidad" (p. 12). Sí que se contempla en la mayor parte de los PPTP, una dotación de espacios para aseos para personas con discapacidad, pero siempre refiriéndose a ellos como "minusválidos".

A partir del estudio de los PCAP, también destaca el hecho de cómo la Administración ha ido otorgando mayor importancia a criterios económicos frente a criterios técnicos a la hora de elegir la propuesta constructiva. Si en el 2009 se otorgaba un peso inferior al $50 \%$ al criterio económico, éste ha ido creciendo hasta llegar al $65 \%$ en 2011 y 2012, para asentarse en el $60 \%$ a partir de 2015. Esto no sólo supone una infravaloración de los aspectos de diseño frente a los meramente monetarios, sino que además, los criterios técnicos que se valoran apenas contemplan cuestiones relativas a la sostenibilidad y a la inclusión. Simplemente se consideran pinceladas relativas a la economía y durabilidad de los materiales, la adaptación al entorno y la adaptabilidad del edificio.

En este sentido, tanto los PPTP como los PCAP apenas hacen referencia a premisas que favorezcan la inclusión y la sostenibilidad. Bien es cierto que en los proyectos más recientes la Administración incluye un nuevo documento con normas de diseño que, aun presentando notables limitaciones en una apuesta decidida a favor de la inclusión y la sostenibilidad, sí recogen un notable número de premisas, principalmente centradas en cuestiones de accesibilidad, energía y materiales.

En definitiva, y aunque se han producido avances significativos en los últimos años, todavía queda mucho camino por andar a fin de convertir los centros educativos gallegos en lugares físicamente más inclusivos y sostenibles.

\section{Referencias}

Bardin, L. (1986). Análisis de contenido. Madrid: Akal.

Booth, T. \& Ainscow, M. (2011). Index for inclusion. Developing learning and participation in schools. Bristol: CSIE

Doménech, J. L. (2004). Huella ecológica y desarrollo sostenible. Madrid: AENOR Ediciones.

Echeita, G. \& Navarro, D. (2014). Educación inclusiva y desarrollo sostenible. Una llamada urgente a pensarlas juntas. Edetania, 46, 141-161.

España. Real Decreto 314/2006, de 17 de marzo, por el que se aprueba el Código Técnico de la Edificación. Boletín Oficial del Estado, 28 de marzo de 2006, núm. 74, pp. 11816-11831.

España. Ley 39/2006, de 14 de diciembre, de Promoción de la Autonomía Personal y Atención a las personas en situación de dependencia. Boletín Oficial del Estado, 15 de diciembre de 2006, núm. 299, pp. 44142-44156.

España. Real Decreto Legislativo 3/2011, de 14 noviembre, por el que se aprueba el texto refundido de la Ley de Contratos del Sector Público. Texto consolidado. Boletín Oficial del Estado, 16 de noviembre de 2011, núm 276, pp. 117729-117914.

European Agency for Development in Special Needs Education (2010). Conclusiones de la conferencia Internacional "La Educación Inclusiva: vía para fomentar la cohesión social". Middelfart: EADSNE.

Galicia. Decreto 35/2000, de 28 de enero, por el que se aprueba el Reglamento de desarrollo y ejecución de la Ley de accesibilidad y supresión de barreras en la Comunidad Autónoma de Galicia. Diario Oficial de 
Galicia, 29 de febrero de 2000, núm. 41, pp. 2652-2702.

González, M. T., Nieto, J. M. \& Portela, A. (2003). Organización y Gestión de Centros Escolares. Dimensiones y procesos. Madrid: Pearson.

Jiménez, M. A. \& García, M. (2013). Diversidad y educación inclusiva: modelos de intervención didáctica. En F. Navaridas (Coord.), Procesos y contextos educativos: nuevas perspectivas para la práctica docente (pp. 197-256). Logroño: Geneuve Ediciones.

López, F. (2002). El análisis de contenido como método de investigación. XXI: Revista de Educación, 4, 167-179.

Parrilla, A., Susinos, T. y Gallego, C. (2014). Landscapes of Inclusion: three Research Contributions to a Theory Of Inclusive Practice. European Conference on Educational Research, Porto, 2014.

Xunta de Galicia (2008-2017). PPTP, PCAP y Normas de diseño y constructivas de los edificios de uso docente. Disponibles en: https://goo.gl/V6miZL 